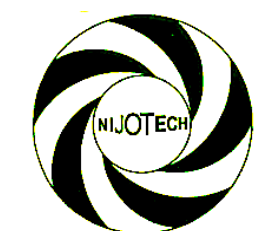

Nigerian Journal of Technology (NIJOTECH)

Vol. 39, No. 1, January 2020, pp. 87 - 96

Copyright(C) Faculty of Engineering, University of Nigeria, Nsukka

Print ISSN: 0331-8443, Electronic ISSN: 2467-8821

www.nijotech.com

http://dx.doi.org/10.4314/njt.v39i1.9

\title{
PREDICTING THE SPLIT TENSILE STRENGTH OF SAW DUST ASH - FINE AGGREGATE CONCRETE
}

\author{
K. M. Oba ${ }^{1, *}$, O. O. Ugwu² and F. O. Okafor ${ }^{3}$ \\ 1, Department of Civil Engineering, Rivers State University, Port Harcourt, Rivers State, NIGERIA \\ 2, Dept of Civil EngR'G, Alex EKWUeme Federal University, Ndufu-Alike IKWo, EBonyi STATE, NIGERIA \\ 3, DePARTMENT OF Civil ENGINEeRING, UNIVERSITY OF NigERIA, NSUKKA, ENUGU STATE, NIGERIA \\ E-mail addresses; 1 kenmieoba@yahoo.com, 2 onuegbuugwu@gmail.com, \\ 3fidelis.okafor@unn.edu.ng
}

\begin{abstract}
The industrial waste, Saw Dust Ash (SDA), has played a key role in concrete mix research. It has served as an alternative or complementary material to some of the traditional materials of concrete. In this study, SDA was used to replace 5\% of the fine aggregate (sand), as the other three ingredients, cement, granite, and water remained constant. Scheffe's simplex lattice was used for five mix ratios in a $\{5,2\}$ component mix, which resulted in additional ten mix ratios. Additional fifteen mix ratios were generated for verification and testing. The thirty concrete mix ratios were subjected to laboratory experiments to determine the 28 days Split Tensile Strengths. The results of the first fifteen Split Tensile strengths were used for the calibration of the model constant coefficients using Scheffe's simplex approach, while those from the second fifteen were used for the model verification. A mathematical regression model was derived from the experimental results, with which the Split Tensile Strengths were predicted. The derived model was subjected to a two-tailed t-test with $5 \%$ significance, which ascertained the model to be adequate with an $R^{2}$ value of 0.8099 . The study revealed that SDA can replace $5 \%$ of fine aggregate and promote sustainability, without compromising the 28 days Split Tensile Strength.
\end{abstract}

Keywords: Saw Dust Ash, Scheffe's simplex lattice, Split Tensile Strength of concrete.

\section{INTRODUCTION}

Industrial waste materials in concrete research and construction has been in use for quite some time. Such materials as fly ash, saw dust ash (SDA), rice husk ash, quarry dust, and palm kernel shell ash are some of the industrial wastes often used in recent times. They have been used in one form or another, to replace fractions of either cement or fine aggregates, while others have been used to stabilise sub-base materials for pavement construction [4].

Saw dust is an industrial waste or bye-product of saw mills produced after the wood has been sawn to shape in a saw mill, and comes out in powder form. It has been used in concrete construction for over 30 years [1]. When saw dust is subjected to fire, it burns to ashes. That ash is called Saw Dust Ash (SDA).
In this study, SDA was used to partially replace $5 \%$ of the fine aggregate. A mathematical model was derived using Scheffe's simplex theory, with which the Split tensile strengths were predicted. There were five components in the mix (water-cement ratio, cement, sand, SDA, and granite).

\section{LITERATURE REVIEW}

The biggest material role in the construction industry, according to [2] is played by concrete. Several authors have studied and determined various means of actualizing sustainability in the construction industry with respect to concrete, using SDA. Ogunribido [3] demonstrated in his experimental research that SDA can drastically improve the properties of lateritic soils when used as a stabiliser. From his findings, the optimum moisture content,

* Corresponding author, tel: +234 8034907294 
maximum dry density, compressive strength, and shear strength of the lateritic soil were improved when stabilised with SDA. A similar study carried out by [4] also shows that using saw dust to stabilise lateritic soils could improve the CBR and other properties of the soil, as well as reduce the construction cost. According to [5], cement is a major source of environmental degradation, as about $400 \mathrm{~kg}$ of $\mathrm{CO}_{2}$ is being emitted for every $600 \mathrm{~kg}$ of cement produced, therefore replacing $10 \%$ of cement with SDA does not negatively affect the chloride permeability and thaw resistance of the concrete, but decreases the drying shrinkage, and increases the water absorption. This also established the pozzolanic ability of SDA. Similarly, [6] found in their research that replacing 5 to $15 \%$ cement content with saw dust increased the compressive, flexural, and split tensile strengths of the concrete for 28days curing period and beyond. It also decreased the weight and cost. However, fewer researchers such as, [7] have carried out research work on replacement of fine aggregates with SDA. Their research findings revealed that $10 \%$ replacement of fine aggregate with SDA will result in an acceptable tensile, flexural, and compressive strengths as well as reduce the amount of wastes in the environment.

SDA has different particles that are mostly angular in shape [7]. According to [7] SDA has a specific gravity of 2.5 , fineness modulus of 1.78 , water absorption of $0.56 \%$, and bulk dry density of $1300 \mathrm{~kg} / \mathrm{m}^{3}$ as against sand with specific gravity of 2.65 , fineness modulus of 2.21 , water absorption of $0.45 \%$, and bulk dry density of $1512 \mathrm{~kg} / \mathrm{m}^{3}$. When $10 \%$ of SDA was added to the sand, these properties became 2.67, 2.2, $0.5 \%$, and $1436 \mathrm{~kg} / \mathrm{m}^{3}$ for specific gravity, fineness modulus, water absorption, and bulk dry density respectively. This significantly indicates that the mixture of sand and 10\% SDA replacement gave similar physical properties with the 0\% SDA replacement, making the mixture adequate for a fine aggregate. However, from the study of [8] SDA had a specific gravity of 2.19 , bulk dry density of $1040 \mathrm{~kg} / \mathrm{m}^{3}$, and moisture content of $0.3 \%$; which gave a bigger difference in the specific gravity of SDA as compared to that of sand. Furthermore, [9] showed that $50 \%$ of the SDA grain size passed through the AASHTO sieve no. $200(75 \mu \mathrm{m})$ while $31 \%$ was retained by sieve no. $325(45 \mu \mathrm{m})$; which justifies the fineness of SDA.

SDA, like many other concrete construction materials, contains several chemical compounds. According to
[7] SDA has the following chemical composition by mass: $65.3 \% \mathrm{SiO}_{2}, 4 \% \mathrm{Al}_{2} \mathrm{O}_{3}, 2.23 \% \mathrm{Fe}_{2} \mathrm{O}_{3}, 9.6 \%$ $\mathrm{CaO}, 5.8 \% \mathrm{MgO}, 0.01 \% \mathrm{MnO}, 0.07 \% \mathrm{Na}_{2} \mathrm{O}, 0.11 \%$ $\mathrm{K}_{2} \mathrm{O}, 0.43 \% \mathrm{P}_{2} \mathrm{O}_{5}$, and $0.45 \% \mathrm{SO}_{2}$. Summing up $\mathrm{SiO}_{2}$, $\mathrm{Al}_{2} \mathrm{O}_{3}$, and $\mathrm{Fe}_{2} \mathrm{O}_{3}$ gives $71.53 \%$. Similar works carried out by [10] reveals $67.95 \% \mathrm{SiO}_{2}, 4.29 \% \mathrm{Al}_{2} \mathrm{O}_{3}$, $2.15 \% \mathrm{Fe}_{2} \mathrm{O}_{3}, 9.47 \% \mathrm{CaO}, 5.84 \% \mathrm{MgO}, 0.01 \% \mathrm{MnO}$, $0.06 \% \mathrm{Na}_{2} \mathrm{O}, 0.11 \% \mathrm{~K}_{2} \mathrm{O}$, and $0.56 \% \mathrm{SO}_{3}$. Summing up $\mathrm{SiO}_{2}, \mathrm{Al}_{2} \mathrm{O}_{3}$, and $\mathrm{Fe}_{2} \mathrm{O}_{3}$ gives 74.39. These, in accordance with [11] indicate that SDA is a good pozzolanic material. The chemical compositions as found by [7]; [8]; [10] all show that SDA has a high percentage of $\mathrm{SiO}_{2}$ and small percentages of $\mathrm{Al}_{2} \mathrm{O}_{3}$ and $\mathrm{Fe}_{2} \mathrm{O}_{3}$, which are similar to those of sand with high percentage of about $95 \% \mathrm{SiO}_{2}$. Hence SDA can be used with sand as fine aggregate.

\subsection{Concrete Mix Design}

There are several methods of concrete mix design. In the United States the American Concrete Institute (ACI) method and the United States Bureau of Reclamation (USBR) method are used. In the United Kingdom and many parts of the world, the Building Research Establishment (BRE) method is used while the Bureau of Indian Standards (BIS) method is used in India.

\subsection{Scheffe's Simplex Theory}

Several authors such as [12]; [13]; [14]; [15]; [16]; [17]; and [18] have carried out concrete mixture research with the development of mathematical models. Most of such works were based on Scheffe's Simplex theory.

Scheffe's model is based on the simplex lattice and simplex theory or approach [19]. The simplex approach considers a number of components, $q$, and a degree of polynomial, $m$. The sum of all the $t^{\text {th }}$ components is not greater than 1 . Hence,

$$
\begin{gathered}
\sum_{i=1}^{q} x_{i}=1 \\
\boldsymbol{x}_{\mathbf{1}}+\boldsymbol{x}_{\mathbf{2}}+\cdots+\boldsymbol{x}_{\boldsymbol{q}}=\mathbf{1}
\end{gathered}
$$

with $0 \leq x \leq 1$. The factor space becomes $S_{q-1 .}$. According to [19] the $\{q, m\}$ simplex lattice design is a symmetrical arrangement of points within the experimental region in a suitable polynomial equation representing the response surface in the simplex region.

The number of points $C_{m}^{(q+m-1)}$ has $(\mathrm{m}+1)$ equally spaced values of $x_{i}=0,1 / \mathrm{m}, 2 / \mathrm{m}, \ldots . \mathrm{m} / \mathrm{m}$. For a $3-$

Vol. 39, No. 1, January 2020 
component mixture with degree of polynomial 2, the corresponding number of points will be $C_{2}^{(3+2-1)}$ which gives 6 (eq. 3 or eq. 4 below) with number of spaced values, $2+1=3$, that is $x_{i}=0,1 / 2$, and 1 and design points of $(1,0,0),(0,1,0),(0,0,1),(1 / 2,1 / 2,0)$, $(1 / 2,01 / 2)$, and $(0,1 / 2,1 / 2)$. Similarly, for a $\{5,2\}$ simplex, there will be 15 points with $x_{i}=0,1 / 2$, and 1 as spaced values. The 15 design points are $(1,0,0,0,0), \quad(0,1,0,0,0), \quad(0,0,1,0,0), \quad(0,0,0,1,0)$, $(0,0,0,0,1), \quad(1 / 2,1 / 2,0,0,0), \quad(1 / 2,0,1 / 2,0,0)$, $(1 / 2,0,0,1 / 2,0), \quad(1 / 2,0,0,0,1 / 2), \quad(0,1 / 2,1 / 2,0,0)$, $(0,0,1 / 2,1 / 2,0), \quad(0,0,0,1 / 2,1 / 2), \quad(0,1 / 2,0,1 / 2,0)$, $(0,0,1 / 2,0,1 / 2),(0,1 / 2,0,0,1 / 2)$.

$$
\begin{aligned}
& N=C_{n}^{(q+n-1)} \\
& \text { or } N=\frac{(q+n-1) !}{(q-1) !(n) !}
\end{aligned}
$$

For a polynomial of degree $m$ with $q$ component variables where eq. (2) holds, the general form is:

$$
\begin{aligned}
Y=b_{0}+\sum b_{i} x_{i}+\sum b_{i j} x_{i} x_{j} \\
+\sum b_{i j k} x_{i} x_{j} x_{k}+\cdots \\
+\sum b_{i 1, i 2 \ldots i n} x_{i 1} x_{i 2} x_{i n}
\end{aligned}
$$

Where $1 \leq i \leq q, 1 \leq i \leq j \leq q, 1 \leq i \leq j \leq k \leq q$, and $b_{0}$ is the constant coefficient.

$x$ is the pseudo component for constituents $i, j$, and $k$.

When $\{q, m\}=\{5,2\}$, eq. (5) becomes:

$Y=b_{0}+b_{1} x_{1}+b_{2} x_{2}+b_{3} x_{3}+b_{4} x_{4}+b_{5} x_{5}+$

$b_{12} x_{1} x_{2}+b_{13} x_{1} x_{3}+b_{14} x_{1} x_{4}+b_{15} x_{1} x_{5}+b_{23} x_{2} x_{3}+$

$b_{24} x_{2} x_{4}+b_{25} x_{2} x_{5}+b_{34} x_{3} x_{4}+b_{35} x_{3} x_{5}+b_{45} x_{4} x_{5}+$

$b_{11} x_{1}^{2}+b_{22} x_{2}^{2}+b_{33} x_{3}^{2}+b_{44} x_{4}^{2}+b_{55} x_{5}^{2}$

and eq. (2) becomes

$x_{1}+x_{2}+x_{3}+x_{4}+x_{5}=1$

Multiplying eq. (7) by $b_{0}$ gives

$b_{0} x_{1}+b_{0} x_{2}+b_{0} x_{3}+b_{0} x_{4}+b_{0} x_{5}=b_{0}$

Multiplying eq. (7) successively by $x_{1}, x_{2}, x_{3}, x_{4}$, and $x_{5}$ and making $x_{1}, x_{2}, x_{3}, x_{4}$, and $x_{5}$ the subjects of the respective formulas:

$x_{1}^{2}=x_{1}-x_{1} x_{2}-x_{1} x_{3}-x_{1} x_{4}-x_{1} x_{5}$

$x_{2}^{2}=x_{2}-x_{1} x_{2}-x_{2} x_{3}-x_{2} x_{4}-x_{2} x_{5}$

$x_{3}^{2}=x_{3}-x_{1} x_{3}-x_{2} x_{3}-x_{3} x_{4}-x_{3} x_{5}$

$x_{4}^{2}=x_{4}-x_{1} x_{4}-x_{2} x_{4}-x_{3} x_{4}-x_{4} x_{5}$

$x_{5}^{2}=x_{5}-x_{1} x_{5}-x_{2} x_{5}-x_{3} x_{5}-x_{4} x_{5}$

Substituting eq. (8) and eq. (9) into eq. (6) we have:

$$
\begin{gathered}
Y=b_{0} x_{1}+b_{0} x_{2}+b_{0} x_{3}+b_{0} x_{4}+b_{0} x_{5}+b_{1} x_{1}+b_{2} x_{2} \\
+b_{3} x_{3}+b_{4} x_{4}+b_{5} x_{5}+b_{12} x_{1} x_{2} \\
+b_{13} x_{1} x_{3}+b_{14} x_{1} x_{4}+b_{15} x_{1} x_{5} \\
+b_{23} x_{2} x_{3}+b_{24} x_{2} x_{4}+b_{25} x_{2} x_{5} \\
+b_{34} x_{3} x_{4}+b_{35} x_{3} x_{5}+b_{45} x_{4} x_{5} \\
+b_{11}\left(x_{1}-x_{1} x_{2}-x_{1} x_{3}-x_{1} x_{4}\right. \\
\left.-x_{1} x_{5}\right)+b_{22}\left(x_{2}-x_{1} x_{2}-x_{2} x_{3}\right. \\
\left.-x_{2} x_{4}-x_{2} x_{5}\right)+b_{33}\left(x_{3}-x_{1} x_{3}\right. \\
\left.-x_{2} x_{3}-x_{3} x_{4}-x_{3} x_{5}\right)+b_{44}\left(x_{4}\right. \\
\left.-x_{1} x_{4}-x_{2} x_{4}-x_{3} x_{4}-x_{4} x_{5}\right) \\
+b_{55}\left(x_{5}-x_{1} x_{5}-x_{2} x_{5}-x_{3} x_{5}\right. \\
\left.-x_{4} x_{5}\right) \\
Y=\left(b_{0}+b_{1}+b_{11}\right) x_{1}+\left(b_{0}+b_{2}+b_{22}\right) x_{2}+\left(b_{0}+\right. \\
\left.b_{3}+b_{33}\right) x_{3}+\left(b_{0}+b_{4}+b_{44}\right) x_{4}+\left(b_{0}+b_{5}+b_{55}\right) x_{5}+ \\
\left(b_{12}-b_{11}-b_{22}\right) x_{1} x_{2}+\left(b_{13}-b_{11}-b_{33}\right) x_{1} x_{3}+ \\
\left(b_{14}-b_{11}-b_{44}\right) x_{1} x_{4}+\left(b_{15}-b_{11}-b_{55}\right) x_{1} x_{5}+ \\
\left(b_{23}-b_{22}-b_{33}\right) x_{2} x_{3}+\left(b_{24}-b_{22}-b_{44}\right) x_{2} x_{4}+ \\
\left(b_{25}-b_{22}-b_{55}\right) x_{2} x_{5}+\left(b_{34}-b_{33}-b_{44}\right) x_{3} x_{4}+ \\
\left(b_{35}-b_{33}-b_{55}\right) x_{3} x_{5}+\left(b_{45}-b_{44}-b_{55}\right) x_{4} x_{5}
\end{gathered}
$$

Let

$$
\begin{aligned}
& \beta_{1}=b_{0}+b_{1}+b_{11} ; \beta_{2}=b_{0}+b_{2}+b_{22} ; \\
& \beta_{3}=b_{0}+b_{3}+b_{33} ; \beta_{4}=b_{0}+b_{4}+b_{44} \\
& \beta_{5}=b_{0}+b_{5}+b_{55} ; \beta_{12}=b_{12}-b_{11}-b_{22} \\
& \beta_{13}=b_{13}-b_{11}-b_{33} ; \beta_{14}=b_{14}-b_{11}-b_{44} \\
& \beta_{15}=b_{15}-b_{11}-b_{55} ; \beta_{23}=b_{23}-b_{22}-b_{33} \\
& \beta_{24}=b_{24}-b_{22}-b_{44} ; \beta_{25}=b_{25}-b_{22}-b_{55} \\
& \beta_{34}=b_{34}-b_{33}-b_{44} ; \beta_{35}=b_{35}-b_{33}-b_{55} \\
& \beta_{45}=b_{45}-b_{44}-b_{55}
\end{aligned}
$$

Substituting eq. (11) into eq. (10) gives

$Y=\beta_{1} x_{1}+\beta_{2} x_{2}+\beta_{3} x_{3}+\beta_{4} x_{4}+\beta_{5} x_{5}+\beta_{12} x_{1} x_{2}+$ $\beta_{13} x_{1} x_{3}+\beta_{14} x_{1} x_{4}+\beta_{15} x_{1} x_{5}+\beta_{23} x_{2} x_{3}+\beta_{24} x_{2} x_{4}+$ $\beta_{25} x_{2} x_{5}+\beta_{34} x_{3} x_{4}+\beta_{35} x_{3} x_{5}+\beta_{45} x_{4} x_{5}$

This can be rewritten as:

$$
Y=\sum_{i=1}^{5} \beta_{i} x_{i}+\sum_{1 \leq i \leq j \leq 5} \beta_{i j} x_{i} x_{j}
$$

Where the response, $\mathrm{Y}$ is a dependent variable (tensile strength of concrete). Hence, eq. (12) is the general equation for a $\{5,2\}$ polynomial, and it has 15 terms, which conforms to Scheffe's theory in eq. (3).

Let $Y_{i}$ denote response to pure components, and $Y_{i j}$ denote response to mixture components in $i$ and $j$. If $x_{i}=1$ and $x_{j}=0$, since $j \neq i$, then

$$
\boldsymbol{Y}_{\boldsymbol{i}}=\boldsymbol{\beta}_{\boldsymbol{i}}
$$

Which means 


$$
\sum_{i=1}^{5} \beta_{i} x_{i}=\sum_{i=1}^{5} Y_{i} x_{i}
$$

Hence, from eq. (14)

$Y_{1}=\beta_{1} ; Y_{2}=\beta_{2} ; Y_{3}=\beta_{3} ; Y_{4}=\beta_{4} ; Y_{5}=\beta_{5}$

According to [19],

Substituting eq. (14)

$$
\beta_{i j}=4 Y_{i j}-2 \beta_{i}-2 \beta_{j}
$$

$$
\beta_{i j}=4 Y_{i j}-2 Y_{i}-2 Y_{j}
$$

\section{MATERIALS AND METHODS}

Water, cement, sand, SDA, and granite were the materials used to produce the concrete.

The first five concrete mix ratios derived from different mix design methods given as

BRE $12=\left[\begin{array}{lllll}0.54 & 1 & 1.9475 & 0.1025 & 2.95\end{array}\right] ;$

BRE $22=\left[\begin{array}{lllll}0.58 & 1 & 2.1185 & 0.1115 & 3.21\end{array}\right] ;$

USBR $22=\left[\begin{array}{lllll}0.58 & 1 & 2.2515 & 0.1185 & 3.29\end{array}\right] ;$

BIS $12=\left[\begin{array}{lllll}0.43 & 1 & 1.2065 & 0.0635 & 2.88\end{array}\right] ;$

ACI $12=\left[\begin{array}{lllll}0.55 & 1 & 1.8335 & 0.0965 & 3.09\end{array}\right]$

These can be transposed and put in matrix form as follows:

$$
S=\left[\begin{array}{ccccc}
0.54 & 0.58 & 0.58 & 0.43 & 0.55 \\
1 & 1 & 1 & 1 & 1 \\
1.9475 & 2.1185 & 2.2515 & 1.2065 & 1.8335 \\
0.1025 & 0.1115 & 0.1185 & 0.0635 & 0.0965 \\
2.95 & 3.21 & 3.29 & 2.88 & 3.09
\end{array}\right] \text { (19) }
$$

Their corresponding pseudo components are given as:

$$
X=\left[\begin{array}{lllll}
1 & 0 & 0 & 0 & 0 \\
0 & 1 & 0 & 0 & 0 \\
0 & 0 & 1 & 0 & 0 \\
0 & 0 & 0 & 1 & 0 \\
0 & 0 & 0 & 0 & 1
\end{array}\right]
$$

With centre points

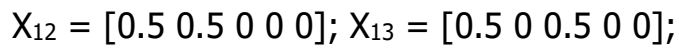

$X_{14}=\left[\begin{array}{lllll}0.5 & 0 & 0 & 0.5 & 0\end{array}\right] ; X_{15}=\left[\begin{array}{lllll}0.5 & 0 & 0 & 0 & 0.5\end{array}\right] ;$

$X_{23}=\left[\begin{array}{lllll}0 & 0.5 & 0.5 & 0 & 0\end{array}\right] ; X_{24}=\left[\begin{array}{lllll}0 & 0.5 & 0 & 0.5 & 0\end{array}\right] ; ;$

$\mathrm{X}_{25}=\left[\begin{array}{lllll}0 & 0.5 & 0 & 0 & 0.5\end{array}\right] ; X_{34}=\left[\begin{array}{lllll}0 & 0 & 0.5 & 0.5 & 0\end{array}\right] ;$

$X_{35}=\left[\begin{array}{lllll}0 & 0 & 0.5 & 0 & 0.5\end{array}\right] ; X_{45}=\left[\begin{array}{lllll}0 & 0 & 0 & 0.5 & 0.5\end{array}\right]$

According to Scheffe (1958),

$$
\mathrm{S}_{\mathrm{ij}}=X \mathrm{~S}_{\mathrm{i}}
$$

Substituting,

$$
\left[\begin{array}{l}
S_{12} \\
S_{13} \\
S_{14} \\
S_{15} \\
S_{23}
\end{array}\right]=\left[\begin{array}{ccccc}
0.5 & 0.5 & 0 & 0 & 0 \\
0.5 & 0 & 0.5 & 0 & 0 \\
0.5 & 0 & 0 & 0.5 & 0 \\
0.5 & 0 & 0 & 0 & 0.5 \\
0 & 0.5 & 0.5 & 0 & 0
\end{array}\right] \times\left[\begin{array}{c}
0.54 \\
0.58 \\
0.58 \\
0.43 \\
0.55
\end{array}\right]
$$

This process is repeated for $S_{24}, S_{25}, S_{34}, S_{35}$, and $S_{45}$. Similarly, this process is repeated for an additional 15 (control) points that will be used for the verification of the formulated model. The regular pentagons for the actual components with their corresponding pseudo components are given in figures (1) and (2) respectively.

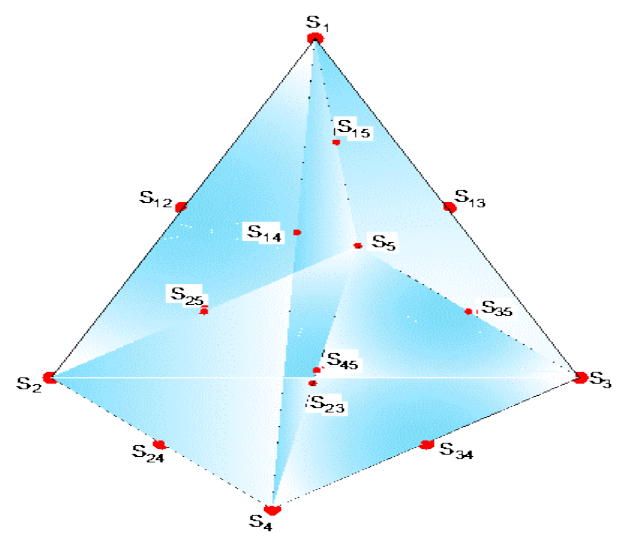

Fig. 1: Simplex Plot for Actual Comp

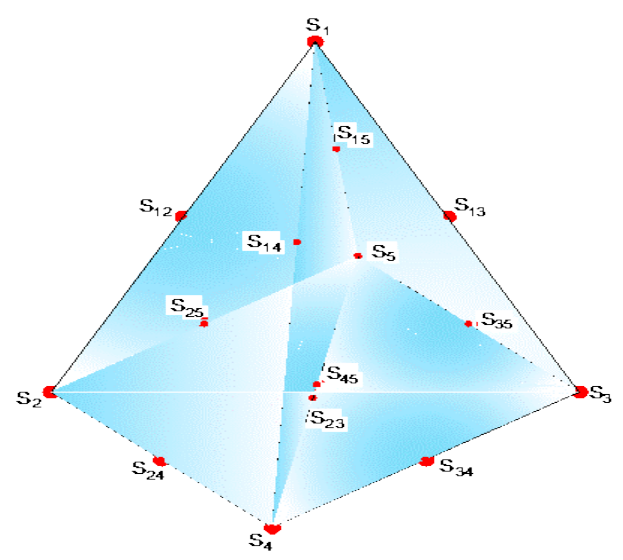

Fig. 2: Simplex Plot for Pseudo Components

\subsection{Split Tensile Test}

The concrete samples were prepared in cylindrical shapes of $300 \mathrm{~mm} \times 150 \mathrm{~mm}$ diameter. The split tensile test which is the most commonly used indirect tensile test was used to determine the tensile strength of the concrete. The specimens were subjected to a compressive load along the vertical diameter at a constant rate. This brought about a tensile split in the specimen. The tensile strength is then determined by,

$$
f_{t}=\frac{2 P}{\pi l d}
$$

Where $\mathrm{P}=$ the load at failure $(\mathrm{KN})$

$\mathrm{d}=$ the diameter of the specimen in millimetres $I=$ the span length of specimen in millimetres Two replicates were made, and the average taken and recorded (see table 4). 
Table 1: Model Mix Ratios

\begin{tabular}{|c|c|c|c|c|c|c|c|c|c|c|c|}
\hline \multirow{3}{*}{$\begin{array}{l}\text { Sample } \\
\text { Points }\end{array}$} & \multicolumn{5}{|c|}{ Actual Components } & \multirow{3}{*}{$\begin{array}{c}\text { Response } \\
\mathbf{Y}_{\exp }\end{array}$} & \multicolumn{5}{|c|}{ Pseudo Components } \\
\hline & w-c ratio & Cement & Sand & SDA & Granite & & w-c ratio & Cement & Sand & SDA & Granite \\
\hline & $\mathrm{S}_{1}$ & $\mathrm{~S}_{2}$ & $\mathrm{~S}_{3}$ & $\mathrm{~S}_{4}$ & $\mathrm{~S}_{5}$ & & $\mathrm{X}_{1}$ & $\mathrm{X}_{2}$ & $\mathrm{X}_{3}$ & $\mathrm{X}_{4}$ & $\mathrm{X}_{5}$ \\
\hline BRE12 & 0.54 & 1 & 1.9475 & 0.1025 & 2.95 & $Y_{1}$ & 1 & 0 & 0 & 0 & 0 \\
\hline BRE22 & 0.58 & 1 & 2.1185 & 0.1115 & 3.21 & $\mathrm{Y}_{2}$ & 0 & 1 & 0 & 0 & 0 \\
\hline USBR22 & 0.58 & 1 & 2.2515 & 0.1185 & 3.29 & $\mathrm{Y}_{3}$ & 0 & 0 & 1 & 0 & 0 \\
\hline BIS12 & 0.43 & 1 & 1.2065 & 0.0635 & 2.88 & $\mathrm{Y}_{4}$ & 0 & 0 & 0 & 1 & 0 \\
\hline ACI12 & 0.55 & 1 & 1.8335 & 0.0965 & 3.09 & $\mathrm{Y}_{5}$ & 0 & 0 & 0 & 0 & 1 \\
\hline N1 & 0.56 & 1 & 2.033 & 0.107 & 3.08 & $\mathrm{Y}_{12}$ & 0.5 & 0.5 & 0 & 0 & 0 \\
\hline $\mathrm{N} 2$ & 0.56 & 1 & 2.0995 & 0.1105 & 3.12 & $\mathrm{Y}_{13}$ & 0.5 & 0 & 0.5 & 0 & 0 \\
\hline N3 & 0.485 & 1 & 1.577 & 0.083 & 2.915 & $\mathrm{Y}_{14}$ & 0.5 & 0 & 0 & 0.5 & 0 \\
\hline $\mathrm{N} 4$ & 0.545 & 1 & 1.8905 & 0.0995 & 3.02 & $Y_{15}$ & 0.5 & 0 & 0 & 0 & 0.5 \\
\hline N5 & 0.58 & 1 & 2.185 & 0.115 & 3.25 & $Y_{23}$ & 0 & 0.5 & 0.5 & 0 & 0 \\
\hline N6 & 0.505 & 1 & 1.6625 & 0.0875 & 3.045 & $Y_{24}$ & 0 & 0.5 & 0 & 0.5 & 0 \\
\hline N7 & 0.565 & 1 & 1.976 & 0.104 & 3.15 & $Y_{25}$ & 0 & 0.5 & 0 & 0 & 0.5 \\
\hline N8 & 0.505 & 1 & $\begin{array}{l}1.729 \\
\end{array}$ & 0.091 & 3.085 & $\mathrm{Y}_{34}$ & 0 & 0 & 0.5 & 0.5 & 0 \\
\hline N9 & 0.565 & 1 & 2.0425 & 0.1075 & 3.19 & $\mathrm{Y}_{35}$ & 0 & 0 & 0.5 & 0 & 0.5 \\
\hline N10 & 0.49 & 1 & 1.52 & 0.08 & 2.985 & $Y_{45}$ & 0 & 0 & 0 & 0.5 & 0.5 \\
\hline
\end{tabular}

Table 2: Control Points

\begin{tabular}{|c|c|c|c|c|c|c|c|c|c|c|c|}
\hline \multirow{3}{*}{$\begin{array}{l}\text { Sample } \\
\text { Points }\end{array}$} & \multicolumn{5}{|c|}{$\begin{array}{c}\text { Actual Components } \\
\end{array}$} & \multirow{3}{*}{$\begin{array}{c}\text { Response } \\
Y_{\exp }\end{array}$} & \multicolumn{5}{|c|}{$\begin{array}{c}\text { Pseudo Components } \\
\end{array}$} \\
\hline & w-c ratio & Cement & Sand & SDA & Granite & & \begin{tabular}{|l|} 
w-c ratio \\
\end{tabular} & Cement & Sand & SDA & Granite \\
\hline & $\mathrm{S}_{1}$ & $\mathrm{~S}_{2}$ & $\mathrm{~S}_{3}$ & $\mathrm{~S}_{4}$ & $\mathrm{~S}_{5}$ & & \begin{tabular}{|l|}
$X_{1}$ \\
\end{tabular} & $\mathrm{X}_{2}$ & $\mathrm{X}_{3}$ & $\mathrm{X}_{4}$ & $\mathrm{X}_{5}$ \\
\hline $\mathrm{C} 1$ & 0.558 & 1 & 2.0463 & 0.1077 & 3.114 & $\mathrm{Y}_{\mathrm{C} 1}$ & 0.4 & 0 & 0.4 & 0 & 0.2 \\
\hline $\mathrm{C} 2$ & 0.52 & 1 & 1.7537 & 0.0923 & 3.078 & $\mathrm{Y}_{\mathrm{C} 2}$ & 0 & 0.6 & 0 & 0.4 & 0 \\
\hline $\mathrm{C} 3$ & 0.548 & 1 & 2.0083 & 0.1057 & 3.018 & $\mathrm{Y}_{\mathrm{C} 3}$ & 0.8 & 0 & 0.2 & 0 & 0 \\
\hline $\mathrm{C} 4$ & 0.49 & 1 & 1.5713 & 0.0827 & 3.012 & $\mathrm{Y}_{\mathrm{C} 4}$ & 0 & 0.4 & 0 & 0.6 & 0 \\
\hline $\mathrm{C} 5$ & 0.544 & 1 & 1.9019 & 0.1001 & 3.006 & $\mathrm{Y}_{\mathrm{C} 5}$ & 0.6 & 0 & 0 & 0 & 0.4 \\
\hline C6 & 0.55 & 1 & 2.0425 & 0.1075 & 3.208 & $\mathrm{Y}_{\mathrm{C} 6}$ & 0 & 0 & 0.8 & 0.2 & 0 \\
\hline $\mathrm{C} 7$ & 0.55 & 1 & 1.9589 & 0.1031 & 3.03 & $\mathrm{Y}_{\mathrm{C} 7}$ & 0.6 & 0.2 & 0 & 0 & 0.2 \\
\hline $\mathrm{C} 8$ & 0.514 & 1 & 1.6967 & 0.0893 & 3.054 & $\mathrm{Y}_{\mathrm{C} 8}$ & 0 & 0.4 & 0 & 0.4 & 0.2 \\
\hline C9 & 0.548 & 1 & 1.8563 & 0.0977 & 3.062 & $\mathrm{Y}_{\mathrm{C} 9}$ & 0.2 & 0 & 0 & 0 & 0.8 \\
\hline C10 & 0.46 & 1 & 1.4155 & 0.0745 & 2.962 & $\mathrm{Y}_{\mathrm{C} 10}$ & 0 & 0 & 0.2 & 0.8 & 0 \\
\hline C11 & 0.566 & 1 & 2.1071 & 0.1109 & 3.182 & $\mathrm{Y}_{\mathrm{C} 11}$ & 0.2 & 0 & 0.6 & 0 & 0.2 \\
\hline $\mathrm{C} 12$ & 0.544 & 1 & 1.9323 & 0.1017 & 3.152 & $\mathrm{Y}_{\mathrm{C} 12}$ & 0 & 0.2 & 0.4 & 0.2 & 0.2 \\
\hline $\mathrm{C} 13$ & 0.58 & 1 & 2.1451 & 0.1129 & 3.226 & $\mathrm{Y}_{\mathrm{C} 13}$ & 0 & 0.8 & 0.2 & 0 & 0 \\
\hline C14 & 0.532 & 1 & 1.7651 & 0.0929 & 3.072 & $\mathrm{Y}_{\mathrm{C} 14}$ & 0 & 0.2 & 0 & 0.2 & 0.6 \\
\hline $\mathrm{C} 15$ & 0.536 & 1 & 1.8715 & 0.0985 & 3.084 & $\mathrm{Y}_{\mathrm{C} 15}$ & 0.2 & 0.2 & 0.2 & 0.2 & 0.2 \\
\hline
\end{tabular}

\section{RESULTS AND DISCUSSIONS}

\subsection{Scheffe's Model for 28 days Split Tensile Strength}

The coefficients of polynomial with the aid of table (4), eq. (16), and eq. (18) are:

$\beta_{1}=2.987, \beta_{2}=2.378, \beta_{3}=2.625, \beta_{4}=3.799$,

$\beta_{5}=3.176, \beta_{12}=4 Y_{12}-2 Y_{1}-2 Y_{2}$

$\beta_{12}=4 \times 3.069-2 \times 2.987-2 \times 2.625=1.546$

Similarly, $\beta_{13}=-2.336, \beta_{14}=-2.836, \beta_{15}=-0.086$, $\beta_{23}=1.418, \beta_{24}=-0.666, \beta_{25}=0.36$, $\beta_{34}=-0.704, \beta_{35}=-2.19, \beta_{45}=-2.714$.

Substituting the above coefficients into eq. (12) gives $Y=2.987 x_{1}+2.378 x_{2}+2.625 x_{3}+3.799 x_{4}+$ $3.176 x_{5}+1.546 x_{1} x_{2}-2.336 x_{1} x_{3}-2.836 x_{1} x_{4}-$ $0.086 x_{1} x_{5}+1.418 x_{2} x_{3}-0.666 x_{2} x_{4}-0.36 x_{2} x_{5}-$ $0.704 x_{3} x_{4}-2.19 x_{3} x_{5}-2.714 x_{4} x_{5}$

Eq. (24) above is the mathematical model to predict the 28 days split tensile strength of concrete using SDA to replace $5 \%$ of fine aggregate. 
Predicting the Split Tensile Strength of Saw Dust Ash - Fine Aggregate Concrete, K. M. Oba, et al

Table 3: Sieve Analysis Data for Fine Aggregate with 5\% SDA replacement

\begin{tabular}{|c|c|c|c|c|c|c|c|}
\hline \multicolumn{3}{|c|}{ Standard Sieve Opening Sizes } & \multirow[b]{2}{*}{$\begin{array}{l}\text { Mass } \\
\text { Retained }(\mathrm{g})\end{array}$} & \multirow[b]{2}{*}{$\begin{array}{l}\text { Cumulative Mass } \\
\text { Retained }(\mathrm{g})\end{array}$} & \multirow[b]{2}{*}{$\begin{array}{l}\% \\
\text { Retained }\end{array}$} & \multirow[b]{2}{*}{$\begin{array}{l}\text { Cumulative \% } \\
\text { Retained }\end{array}$} & \multirow[b]{2}{*}{$\begin{array}{l}\% \\
\text { Passing }\end{array}$} \\
\hline $\begin{array}{l}\text { Sieve } \\
\text { Number }\end{array}$ & $\begin{array}{l}\text { Sieve size } \\
\text { (in) }\end{array}$ & $\begin{array}{l}\text { Sieve size } \\
(\mathrm{mm})\end{array}$ & & & & & \\
\hline $1 / 4^{\prime \prime}$ & 0.25 & 6.3 & 0 & 0 & $0.00 \%$ & $0.00 \%$ & $100.00 \%$ \\
\hline$\# 4$ & 0.187 & 4.75 & 2.2 & 2.2 & $0.73 \%$ & $0.73 \%$ & $99.27 \%$ \\
\hline \#8 & 0.0929 & 2.36 & 10.6 & 12.8 & $3.51 \%$ & $4.24 \%$ & $95.76 \%$ \\
\hline \#16 & 0.0465 & 1.18 & 29.7 & 42.5 & $9.84 \%$ & $14.08 \%$ & $85.92 \%$ \\
\hline \#30 & 0.0236 & 0.6 & 89.1 & 131.6 & $29.52 \%$ & $43.61 \%$ & $56.39 \%$ \\
\hline$\# 50$ & 0.0118 & 0.3 & 111.3 & 242.9 & $36.88 \%$ & $80.48 \%$ & $19.52 \%$ \\
\hline \#100 & 0.00591 & 0.15 & 52.5 & 295.4 & $17.40 \%$ & $97.88 \%$ & $2.12 \%$ \\
\hline \#200 & 0.00295 & 0.075 & 6.1 & 301.5 & $2.02 \%$ & $99.90 \%$ & $0.10 \%$ \\
\hline \multirow[t]{2}{*}{ Pan } & 0 & 0 & 0.3 & 301.8 & $0.10 \%$ & $100.00 \%$ & \\
\hline & & Total mass & 301.8 & & & & \\
\hline
\end{tabular}

Fineness modulus, $F M=\frac{0.73+4.24+14.08+43.61+80.48+97.88}{100}=2.41$

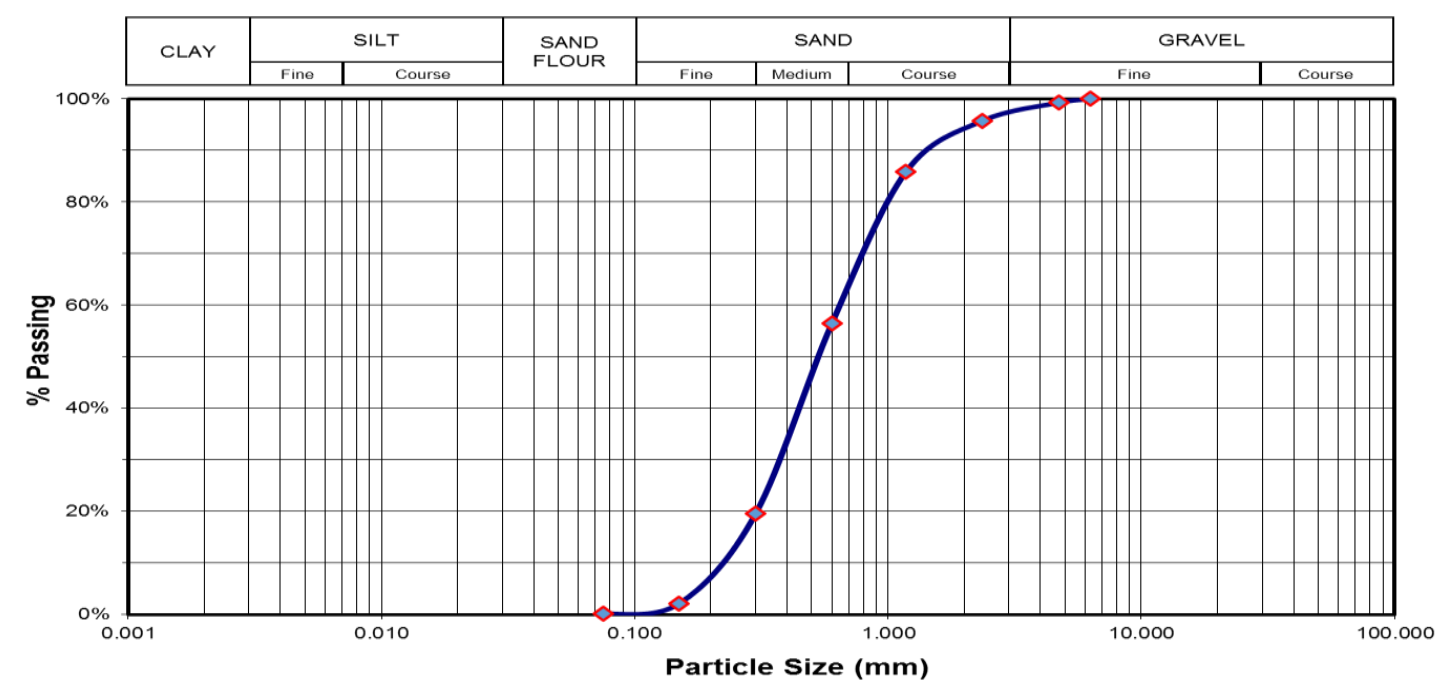

Fig. 3: Particle Size Distribution for Fine Aggregate with 5\% SDA replacement

Table 4: Split Tensile Strength of Concrete

\begin{tabular}{|c|c|c|c|c|c|c|c|}
\hline \multirow{2}{*}{ Sample } & \multirow{2}{*}{ Curing } & \multicolumn{2}{|c|}{ Load (KN) } & \multirow{2}{*}{$\frac{2}{\pi L d}$} & \multicolumn{3}{|c|}{ Split tensile Strength $\left(\mathrm{N} / \mathrm{mm}^{2}\right)$} \\
\hline & & A & B & & A & B & Average \\
\hline \multirow{2}{*}{ BRE12 } & 7 Days & 145.96 & 179.82 & 14.1471 & 2.065 & 2.544 & 2.304 \\
\hline & 28 Days & 221.17 & 201.06 & 14.1471 & 3.129 & 2.844 & 2.987 \\
\hline \multirow{2}{*}{ BRE22 } & 7 Days & 165.88 & 165.73 & 14.1471 & 2.347 & 2.345 & 2.346 \\
\hline & 28 Days & 163.36 & 172.79 & 14.1471 & 2.311 & 2.444 & 2.378 \\
\hline \multirow{2}{*}{ USBR22 } & 7 Days & 121.58 & 152.34 & 14.1471 & 1.720 & 2.155 & 1.938 \\
\hline & 28 Days & 185.13 & 186 & 14.1471 & 2.619 & 2.631 & 2.625 \\
\hline \multirow{2}{*}{ BIS12 } & 7 Days & 203.43 & 203.43 & 14.1471 & 2.878 & 2.878 & 2.878 \\
\hline & 28 Days & 284.2 & 252.9 & 14.1471 & 4.021 & 3.578 & 3.799 \\
\hline \multirow{2}{*}{ ACI12 } & 7 Days & 191.97 & 195.87 & 14.1471 & 2.716 & 2.771 & 2.743 \\
\hline & 28 Days & 223.12 & 225.88 & 14.1471 & 3.157 & 3.196 & 3.176 \\
\hline \multirow{2}{*}{ N1 } & 7 Days & 188.81 & 169.65 & 14.1471 & 2.671 & 2.400 & 2.536 \\
\hline & 28 Days & 207.35 & 226.55 & 14.1471 & 2.933 & 3.205 & 3.069 \\
\hline \multirow{2}{*}{ N2 } & 7 Days & 150.76 & 138.23 & 14.1471 & 2.133 & 1.956 & 2.044 \\
\hline & 28 Days & 155.19 & 158.96 & 14.1471 & 2.195 & 2.249 & 2.222 \\
\hline \multirow{2}{*}{ N3 } & 7 Days & 141.37 & 186.23 & 14.1471 & 2.000 & 2.635 & 2.317 \\
\hline & 28 Days & 189.06 & 190.38 & 14.1471 & 2.675 & 2.693 & 2.684 \\
\hline \multirow{2}{*}{ N4 } & 7 Days & 154.57 & 156.45 & 14.1471 & 2.187 & 2.213 & 2.200 \\
\hline & 28 Days & 222.42 & 210.17 & 14.1471 & 3.147 & 2.973 & 3.060 \\
\hline
\end{tabular}




\begin{tabular}{|c|c|c|c|c|c|c|c|}
\hline \multirow{2}{*}{ Sample } & \multirow{2}{*}{ Curing } & \multicolumn{2}{|c|}{ Load (KN) } & \multirow{2}{*}{$\frac{2}{\pi L d}$} & \multicolumn{3}{|c|}{ Split tensile Strength $\left(\mathrm{N} / \mathrm{mm}^{2}\right)$} \\
\hline & & A & B & & $\mathrm{A}$ & $\mathrm{B}$ & Average \\
\hline \multirow{2}{*}{ N5 } & 7 Days & 165.88 & 182.65 & 14.1471 & 2.347 & 2.584 & 2.465 \\
\hline & 28 Days & 207.66 & 196.04 & 14.1471 & 2.938 & 2.773 & 2.856 \\
\hline \multirow{2}{*}{ N6 } & 7 Days & 132.26 & 165.25 & 14.1471 & 1.871 & 2.338 & 2.104 \\
\hline & 28 Days & 204.83 & 208.29 & 14.1471 & 2.898 & 2.947 & 2.922 \\
\hline \multirow{2}{*}{ N7 } & 7 Days & 144.2 & 173.1 & 14.1471 & 2.040 & 2.449 & 2.244 \\
\hline & 28 Days & 199.84 & 205.46 & 14.1471 & 2.827 & 2.907 & 2.867 \\
\hline \multirow{2}{*}{ N8 } & 7 Days & 169.33 & 148.27 & 14.1471 & 2.396 & 2.098 & 2.247 \\
\hline & 28 Days & 219.28 & 209.86 & 14.1471 & 3.102 & 2.969 & 3.036 \\
\hline \multirow{2}{*}{ N9 } & 7 Days & 125.05 & 182.38 & 14.1471 & 1.769 & 2.580 & 2.175 \\
\hline & 28 Days & 158.56 & 174.04 & 14.1471 & 2.243 & 2.462 & 2.353 \\
\hline \multirow{2}{*}{ N10 } & 7 Days & 138.86 & 172.11 & 14.1471 & 1.964 & 2.435 & 2.200 \\
\hline & 28 Days & 199.49 & 197.61 & 14.1471 & 2.822 & 2.796 & 2.809 \\
\hline \multirow{2}{*}{$\mathrm{C} 1$} & 7 Days & 139.8 & 157.93 & 14.1471 & 1.978 & 2.234 & 2.106 \\
\hline & 28 Days & 170.3 & 172.1 & 14.1471 & 2.409 & 2.435 & 2.422 \\
\hline \multirow{2}{*}{ C2 } & 7 Days & 139.96 & 145.33 & 14.1471 & 1.980 & 2.056 & 2.018 \\
\hline & 28 Days & 189.66 & 189.5 & 14.1471 & 2.683 & 2.681 & 2.682 \\
\hline \multirow{2}{*}{ C3 } & 7 Days & 151.45 & 155.19 & 14.1471 & 2.143 & 2.195 & 2.169 \\
\hline & 28 Days & 182 & 183.31 & 14.1471 & 2.575 & 2.593 & 2.584 \\
\hline \multirow{2}{*}{ C4 } & 7 Days & 140.75 & 152.46 & 14.1471 & 1.991 & 2.157 & 2.074 \\
\hline & 28 Days & 219.91 & 226.51 & 14.1471 & 3.111 & 3.204 & 3.158 \\
\hline \multirow{2}{*}{ C5 } & 7 Days & 157.39 & 157.08 & 14.1471 & 2.227 & 2.222 & 2.224 \\
\hline & 28 Days & 204.2 & 200.75 & 14.1471 & 2.889 & 2.840 & 2.864 \\
\hline \multirow{2}{*}{ C6 } & 7 Days & 140.64 & 148.04 & 14.1471 & 1.990 & 2.094 & 2.042 \\
\hline & 28 Days & 201.47 & 201 & 14.1471 & 2.850 & 2.844 & 2.847 \\
\hline \multirow{2}{*}{ C7 } & 7 Days & 153.94 & 166.22 & 14.1471 & 2.178 & 2.352 & 2.265 \\
\hline & 28 Days & 197.92 & 202.63 & 14.1471 & 2.800 & 2.867 & 2.833 \\
\hline \multirow{2}{*}{ C8 } & 7 Days & 146.12 & 148.5 & 14.1471 & 2.067 & 2.101 & 2.084 \\
\hline & 28 Days & 218.34 & 216.77 & 14.1471 & 3.089 & 3.067 & 3.078 \\
\hline \multirow{2}{*}{ C9 } & 7 Days & 157.08 & 186.6 & 14.1471 & 2.222 & 2.640 & 2.431 \\
\hline & 28 Days & 211.78 & 210.5 & 14.1471 & 2.996 & 2.978 & 2.987 \\
\hline \multirow{2}{*}{ C10 } & 7 Days & 190.68 & 183.08 & 14.1471 & 2.698 & 2.590 & 2.644 \\
\hline & 28 Days & 243.97 & 248 & 14.1471 & 3.451 & 3.508 & 3.480 \\
\hline \multirow{2}{*}{ C11 } & 7 Days & 157.08 & 153.62 & 14.1471 & 2.222 & 2.173 & 2.198 \\
\hline & 28 Days & 163.99 & 161.79 & 14.1471 & 2.320 & 2.289 & 2.304 \\
\hline \multirow{2}{*}{ C12 } & 7 Days & 149.23 & 156.45 & 14.1471 & 2.111 & 2.213 & 2.162 \\
\hline & 28 Days & 190.29 & 195.8 & 14.1471 & 2.692 & 2.770 & 2.731 \\
\hline 012 & 7 Days & 180 & 184.32 & 14.1471 & 2.546 & 2.608 & 2.577 \\
\hline IJ & 28 Days & 184.73 & 178.76 & 14.1471 & 2.613 & 2.529 & 2.571 \\
\hline r14 & 7 Days & 157.9 & 157.5 & 14.1471 & 2.234 & 2.228 & 2.231 \\
\hline 14 & 28 Days & 208.3 & 206.34 & 14.1471 & 2.947 & 2.919 & 2.933 \\
\hline C15 & 7 Days & 161.2 & 162.12 & 14.1471 & 2.281 & 2.294 & 2.287 \\
\hline 113 & 28 Days & 198.58 & 196.7 & 14.1471 & 2.809 & 2.783 & 2.796 \\
\hline
\end{tabular}

\subsection{Test of Adequacy of the Model}

A two-tailed student t-test was carried out at $95 \%$ confidence level, which implies $100-95=5 \%$ significance. Since it is a two-tailed, significance $=$ $5 / 2=2.5 \%$

Hence significance level $=100-2.5=97.5 \%$

Let $D$ be difference between the experimental and predicted responses
The mean of the difference, $D_{a}=$ $\frac{1}{n} \sum_{i=1}^{n} D_{i}$

The variance of the difference,

$$
S^{2}=\left(\frac{1}{n-1}\right) \sum_{i=1}^{n}\left(D-D_{a}\right)^{2}{ }_{i}
$$


Predicting the Split Tensile Strength of Saw Dust Ash - Fine Aggregate Concrete, K. M. Oba, et al

$$
t_{\text {calculated }}=\frac{D_{a} \sqrt{n}}{S}
$$

$$
S^{2}=\frac{0.266}{15-1} ; \text { or } S=\sqrt{0.019}=0.138
$$

Where $\mathrm{n}=$ number of observations with degree of freedom $\mathrm{n}-1$.

$$
t_{\text {calculated }}=0.344
$$

\begin{tabular}{|c|c|c|c|c|c|c|c|c|}
\hline \multirow{3}{*}{$\begin{array}{l}\text { Sample } \\
\text { Points }\end{array}$} & \multirow{3}{*}{$\begin{array}{c}\text { Response } \\
\text { Y }\end{array}$} & \multicolumn{5}{|c|}{ Pseudo Components } & \multirow{3}{*}{\begin{tabular}{|c|} 
Split tensile \\
strength \\
$Y_{\text {exp }}\left(\mathrm{N} / \mathrm{mm}^{2}\right)$ \\
\end{tabular}} & \multirow{3}{*}{$\begin{array}{c}\text { Split tensile } \\
\text { strength } \\
Y_{\text {pred }}\left(\mathrm{N} / \mathrm{mm}^{2}\right)\end{array}$} \\
\hline & & W-c ratio & Cement & Sand & SDA & Granite & & \\
\hline & & $\mathrm{X}_{1}$ & $\mathrm{X}_{2}$ & $\mathrm{X}_{3}$ & $\mathrm{X}_{4}$ & $\mathrm{X}_{5}$ & & \\
\hline BRE12 & $\mathrm{Y}_{1}$ & 1 & 0 & 0 & 0 & 0 & 2.987 & 2.987 \\
\hline BRE22 & $\mathrm{Y}_{2}$ & 0 & 1 & 0 & 0 & 0 & 2.378 & 2.378 \\
\hline USBR22 & $\mathrm{Y}_{3}$ & 0 & 0 & 1 & 0 & 0 & 2.625 & 2.625 \\
\hline BIS12 & $\mathrm{Y}_{4}$ & 0 & 0 & 0 & 1 & 0 & 3.799 & 3.799 \\
\hline ACI12 & $\mathrm{Y}_{5}$ & 0 & 0 & 0 & 0 & 1 & 3.176 & 3.176 \\
\hline $\mathrm{N} 1$ & $\mathrm{Y}_{12}$ & 0.5 & 0.5 & 0 & 0 & 0 & 3.069 & 3.069 \\
\hline $\mathrm{N} 2$ & $Y_{13}$ & 0.5 & 0 & 0.5 & 0 & 0 & 2.222 & 2.222 \\
\hline N3 & $\mathrm{Y}_{14}$ & 0.5 & 0 & 0 & 0.5 & 0 & 2.684 & 2.684 \\
\hline N4 & $Y_{15}$ & 0.5 & 0 & 0 & 0 & 0.5 & 3.060 & 3.060 \\
\hline N5 & $Y_{23}$ & 0 & 0.5 & 0.5 & 0 & 0 & 2.856 & 2.856 \\
\hline N6 & $Y_{24}$ & 0 & 0.5 & 0 & 0.5 & 0 & 2.922 & 2.922 \\
\hline N7 & $Y_{25}$ & 0 & 0.5 & 0 & 0 & 0.5 & 2.867 & 2.867 \\
\hline N8 & $Y_{34}$ & 0 & 0 & 0.5 & 0.5 & 0 & 3.036 & 3.036 \\
\hline N9 & $Y_{35}$ & 0 & 0 & 0.5 & 0 & 0.5 & 2.353 & 2.353 \\
\hline N10 & $Y_{45}$ & 0 & 0 & 0 & 0.5 & 0.5 & 2.809 & 2.809 \\
\hline $\mathrm{C} 1$ & $\mathrm{Y}_{\mathrm{C} 1}$ & 0.4 & 0 & 0.4 & 0 & 0.2 & 2.422 & 2.324 \\
\hline $\mathrm{C} 2$ & $\mathrm{Y}_{\mathrm{C} 2}$ & 0 & 0.6 & 0 & 0.4 & 0 & 2.682 & 2.787 \\
\hline C3 & $\mathrm{Y}_{\mathrm{C} 3}$ & 0.8 & 0 & 0.2 & 0 & 0 & 2.584 & 2.541 \\
\hline $\mathrm{C} 4$ & $Y_{\mathrm{C} 4}$ & 0 & 0.4 & 0 & 0.6 & 0 & 3.158 & 3.071 \\
\hline $\mathrm{C} 5$ & $\mathrm{Y}_{\mathrm{C} 5}$ & 0.6 & 0 & 0 & 0 & 0.4 & 2.864 & 3.042 \\
\hline C6 & $\mathrm{Y}_{\mathrm{C} 6}$ & 0 & 0 & 0.8 & 0.2 & 0 & 2.847 & 2.747 \\
\hline $\mathrm{C} 7$ & $\mathrm{Y}_{\mathrm{C} 7}$ & 0.6 & 0.2 & 0 & 0 & 0.2 & 2.833 & 3.093 \\
\hline $\mathrm{C} 8$ & $\mathrm{Y}_{\mathrm{C} 8}$ & 0 & 0.4 & 0 & 0.4 & 0.2 & 3.078 & 2.811 \\
\hline C9 & $\mathrm{Y}_{\mathrm{C} 9}$ & 0.2 & 0 & 0 & 0 & 0.8 & 2.987 & 3.124 \\
\hline $\mathrm{C} 10$ & $\mathrm{Y}_{\mathrm{C} 10}$ & 0 & 0 & 0.2 & 0.8 & 0 & 3.480 & 3.452 \\
\hline C11 & $\mathrm{Y}_{\mathrm{C} 11}$ & 0.2 & 0 & 0.6 & 0 & 0.2 & 2.304 & 2.261 \\
\hline $\mathrm{C} 12$ & $\mathrm{Y}_{\mathrm{C} 12}$ & 0 & 0.2 & 0.4 & 0.2 & 0.2 & 2.731 & 2.682 \\
\hline C13 & $\mathrm{Y}_{\mathrm{C} 13}$ & 0 & 0.8 & 0.2 & 0 & 0 & 2.571 & 2.654 \\
\hline C14 & $\mathrm{Y}_{\mathrm{C} 14}$ & 0 & 0.2 & 0 & 0.2 & 0.6 & 2.933 & 2.832 \\
\hline $\mathrm{C} 15$ & $\mathrm{Y}_{\mathrm{C} 15}$ & 0.2 & 0.2 & 0.2 & 0.2 & 0.2 & 2.796 & 2.665 \\
\hline
\end{tabular}

Table 5: Experimental and predicted values of 28days Split Tensile strength of Concrete 
Predicting the Split Tensile Strength of Saw Dust Ash - Fine Aggregate Concrete, K. M. Oba, et al

Table 6: Student t-test for 28days Split tensile strength of Concrete

\begin{tabular}{|c|c|c|c|c|c|c|}
\hline \multirow{2}{*}{ Sample } & \multirow{2}{*}{ Curing } & \multicolumn{2}{|c|}{ Split tensile Strength $\left(\mathrm{N} / \mathrm{mm}^{2}\right)$} & \multicolumn{3}{|l|}{ t-test } \\
\hline & & Yexperimental & $\mathrm{Y}_{\text {predicted }}$ & $D=Y_{\text {exp }}-Y_{\text {pred }}$ & $D_{a}-D$ & $\left(D_{a}-D\right)^{2}$ \\
\hline $\mathrm{C} 1$ & 28 Days & 2.422 & 2.324 & 0.098 & -0.086 & 0.007 \\
\hline $\mathrm{C} 2$ & 28 Days & 2.682 & 2.787 & -0.105 & 0.117 & 0.014 \\
\hline C3 & 28 Days & 2.584 & 2.541 & 0.043 & -0.031 & 0.001 \\
\hline C4 & 28 Days & 3.158 & 3.071 & 0.087 & -0.075 & 0.006 \\
\hline C5 & 28 Days & 2.864 & 3.042 & -0.178 & 0.190 & 0.036 \\
\hline C6 & 28 Days & 2.847 & 2.747 & 0.100 & -0.088 & 0.008 \\
\hline C7 & 28 Days & 2.833 & 3.093 & -0.260 & 0.272 & 0.074 \\
\hline $\mathrm{C} 8$ & 28 Days & 3.078 & 2.811 & 0.267 & -0.255 & 0.065 \\
\hline C9 & 28 Days & 2.987 & 3.124 & -0.137 & 0.149 & 0.022 \\
\hline C10 & 28 Days & 3.480 & 3.452 & 0.028 & -0.016 & 0.000 \\
\hline C11 & 28 Days & 2.304 & 2.261 & 0.043 & -0.031 & 0.001 \\
\hline C12 & 28 Days & 2.731 & 2.682 & 0.049 & -0.037 & 0.001 \\
\hline C13 & 28 Days & 2.571 & 2.654 & -0.083 & 0.095 & 0.009 \\
\hline C14 & 28 Days & 2.933 & 2.832 & 0.101 & -0.089 & 0.008 \\
\hline C15 & 28 Days & 2.796 & 2.665 & 0.131 & -0.119 & 0.014 \\
\hline \multicolumn{4}{|c|}{ TOTAL } & 0.184 & & 0.266 \\
\hline \multicolumn{4}{|c|}{ AVERAGE $\mathrm{Da}_{\mathrm{a}}$} & 0.012 & & \\
\hline
\end{tabular}

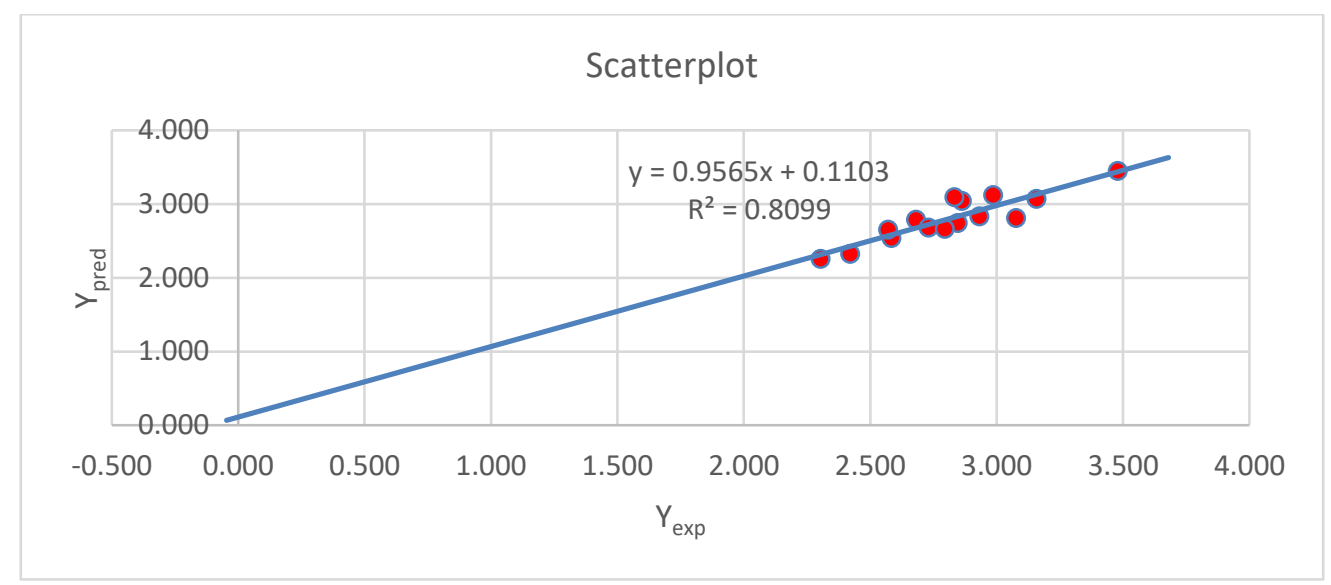

Fig. 4: Scatterplot of Predicted vs. Experimental 28days Split Tensile Strength

From the t-table, $t_{(\beta, v)}$ can be determined where $v=$ $15-1=14$, and $\beta=$ significance level. $t_{(0.975,14)}=$ 2.145

Since $t_{\text {calculated }}(0.344)<\mathrm{t}_{(0.975,14)}(2.145)$, and lies between -2.145 and 2.145 , therefore there is no significant difference between the experimental and predicted responses, $\mathrm{H}_{0}$ is accepted, and $\mathrm{H}_{a}$ is rejected. The model is confirmed to be adequate.

The $R^{2}$ value of 0.8099 indicates that the experimental results are highly correlated to the predicted results. This is also an indication that the model is fit and adequate.

\section{CONCLUSIONS AND RECOMMENDATIONS}

Replacement of fine aggregate with 5\% SDA has resulted in acceptable 28 days Split Tensile strengths (between 2.2 and $3.8 \mathrm{~N} / \mathrm{mm}^{2}$ ) with concrete mix ratios resulting from different design methods. A regression model has been generated from the resulting laboratory experiments using Scheffe's simplex theory. A two-tailed t-test was carried out, which confirmed the adequacy of the derived model with an $R^{2}$ value of 0.8099 . The results also confirmed that SDA is a suitable material to replace a small fraction of fine aggregate in a bid to promote sustainability.

\section{REFERENCES}

[1] C. Marthong, "Sawdust Ash ( SDA ) as Partial Replacement of Cement," Int. J. Eng. Res. Appl., vol. 2, no. 4, pp. 1980-1985, 2012.

[2] M. S. Mamlouk and J. P. Zaniewski, "Materials for Civil and Construction Engineers," 2nd ed., New Jersey: Pearson Prentice Hall, 2006.

[3] T. H. Ogunribido, "Geotechnical Properties of Saw Dust Ash Stabilised South-Western Nigerian 
Lateritic Soils," Environ. Res. Eng. Manag., vol. 2, no. 60, pp. 29-30, 2012.

[4] G. R. Otoko and B. K. Honest, "Stabilization of Nigerian Deltaic Laterites with Saw Dust Ash," Int. J. Sci. Res. Manag., vol. 2, no. 8, pp. 12871292, 2014.

[5] S. Chowdhury, M. Mishra, and O. Suganya, "The incorporation of wood waste ash as a partial cement replacement material for making structural grade concrete: An overview," Ain Shams Eng. J., vol. 6, pp. 429-437, 2015.

[6] A. Sofi, A. Saxena, P. Agrawal, A. R. Sharma, and K. Sharma, "Strength Predictions of Saw Dust and Steel Fibres in Concrete," Int. J. Innov. Res. Sci. Eng. Technol., vol. 4, no. 2, pp. 1247312477, 2015.

[7] M. Mageswari and B. Vidivelli, "The use of sawdust ash as fine aggregate replacement in concrete," J. Environ. Res. Dev., vol. 3, no. 3, pp. 720-726, 2009.

[8] A. A. Raheem, B. S. Olasunkanmi, and C. S. Folorunso, "Saw Dust Ash as Partial Replacement for Cement in Concrete," Organ. Technol. Manag. - An Int. J., vol. 4, no. 2, pp. 474-480, 2012.

[9] T. R. Naik, "Tests of Wood Ash as a Potential Source for Construction Materials," UWM Center for By-product Utilisation, Report No. CBU-199909, Department of Civil Engineering and Mechanics, University of Wisconsin-Milwauke, Milwauke, 1999.

[10] S. T. Tyagher, J. T. Utsev, and T. Adagba, "suitability of saw dust ash-lime mixture for production of sandcrete hollow blocks," Niger. J. Technol., vol. 30, no. 1, pp. 1-6, 2011.

[11] ASTM.C618, Standard Specification for Coal Fly Ash and Raw or Calcined Natural Pozzolan for Use as a Mineral Admixture in Concrete. West
Conshohocken: American Society for Testing and Materials, 2000.

[12] C. U. Anya, "Models for Predicting the Structural Characteristics of Sand-Quarry Dust Blocks," Ph.D Thesis, University of Nigeria, Nsukka, 2015.

[13] Y. M. Gamil and I. H. Bakar, "The Development of Mathematical Prediction Model to Predict Resilient Modulus for Natural Soil Stabilised by POFA-OPC Additive for the Use in Unpaved Road Design," in Soft Soil Engineering International Conference, 2015, pp. 1-11.

[14] P. N. Onuamah, "Prediction-of-the-Compressive -Strength-of-Concrete-with-Palm-KernelAggregate-Using-the-Artificial-NeutralNetworks-Approach.docx," Int. J. Sci. Eng. Res., vol. 6, no. 6, pp. 962-969, 2015.

[15] E. M. Mbadike and N. N. Osadere, "Five Component Concrete Mix Optimization of Aluminum Waste Using Scheffe's Theory," Int. J. Comput. Eng. Res., vol. 4, no. 4, pp. 23-31, 2014.

[16] E. M. Mbadike and N. N. Osadebe, "Application of Scheffe's model in optimization of compressive strength of lateritic concrete," J. Civ. Eng. Constr. Technol., vol. 4, no. 9, pp. 265-274, 2013.

[17] C. E. Okere, D. O. Onwuka, S. U. Onwuka, and J. I. Arimanwa, "Simplex-Based Concrete Mix Design," IOSR J. Mech. Civ. Eng., vol. 5, no. 2, pp. 46-55, 2013.

[18] N. N. Osadebe, C. C. Mbajiorgu, and T. U. Nwakonobi, "An optimization model development for laterized- concrete mix proportioning in building," Niger. J. Technol., vol. 26, no. 1, pp. 37-46, 2007.

[19] H. Scheffe, "Experiments with Mixtures," J. $R$. Stat. Ser. B, vol. 25, no. 2, pp. 235-263, 1958. 